\title{
Ambientes innovadores en México: el caso del Corredor Industrial El Bajío ${ }^{1}$
}

\author{
María del Pilar Monserrat Pérez Hernández²
}

\begin{abstract}
RESUMEN
Las regiones se han transformado de huéspedes pasivos a estructuras activas con capacidad de generar procesos innovadores locales. Este estudio se centra en el caso del Corredor Industrial El Bajío en México, para determinar si cuenta con las características de un ambiente innovador. A partir de un análisis cualitativo, se revisó la literatura y se realizaron una serie de entrevistas a actores que participan en esta actividad innovadora en la región de estudio. Los resultados indicaron que el corredor se fue creando a partir de su ubicación geográfica privilegiada, sin una intencionalidad clara, pero debido a la necesidad de atraer inversiones mayores se diseñó e implemento un proceso de gobernanza local, que está en progreso, donde convergen estrategias de fomento a la innovación diferenciadas.
\end{abstract}

Palabras clave: Geografía de la innovación, ambientes innovadores, proximidad, región, México.

\begin{abstract}
Several regions have been transformed from passive locations to active structures capable of generating and promoting local innovative processes. The analysis presented is centered on the case of the "el Bajio" industrial corridor in Mexico. The goal is to determine if this corridor has the characteristics of an innovative environment. Based on qualitative analysis, we performed a literature review, followed by several interviews with participants in the innovative activities within the study region. The results show that this industrial corridor was created because of its strategic geographic location in order to attract more investments in the region, but without a definitive plan. Therefore, a local regulation process was designed and implemented, yet is still in under revision. This regulation incorporates several strategies aimed at promoting innovation, which the government will support if various states in the region and adopt similar development patterns.
\end{abstract}

Key words: Innovative milieu, proximity, region, Mexico

\footnotetext{
1 Se agradece el apoyo del Instituto Politécnico Nacional por el financiamiento de este trabajo, así como la elaboración de los mapas a Fermín Alí Cruz. Artículo recibido el 23 de marzo de 2015, aceptado el 23 de junio y corregido el 3 de junio de 2015.
}

\footnotetext{
2 Centro de Investigaciones Económicas, Administrativas y Sociales, Instituto Politécnico Nacional (México). E-mail: mpperez@ipn.mx
} 
En la actualidad el conocimiento se ha convertido en un factor de producción clave del crecimiento económico. La acelerada e inédita velocidad con la que la tecnología es creada, apropiada, acumulada y es partícipe de la generación de riqueza, propicia el tránsito acelerado hacia una sociedad basada en el conocimiento. Este nuevo tipo de sociedad requiere acceder al conocimiento en forma creciente, no solo para generar innovaciones en productos y procesos, sino también para incorporar y difundir los saberes originados en otras partes del mundo (David \& Foray, 2001).

Empero, la innovación es un fenómeno selectivo que tiende a concentrarse en ciertas ramas de actividad, en un número limitado de empresas $y$, sobre todo, en espacios concretos, con características que favorecen la generación de iniciativas innovadoras y su difusión en el sistema económico y social (Storper, 1992; Méndez, 1997).

La globalización implica una creciente vulnerabilidad de los territorios, la posición exitosa en la inserción de las cadenas globales es relativa, ya que están sometidas a riesgos e incertidumbre debido a la volatilidad de las ventajas competitivas. La relocalización de numerosas empresas en nuevas áreas situadas en metrópolis cada vez más alejadas de la capital, transforma un territorio tradicionalmente mononuclear en otro policéntrico (Méndez, 2012)

Las aglomeraciones metropolitanas permiten analizar e interpretar las tendencias y contradicciones asociadas al actual estadío de la globalización. Los cambios en las principales aglomeraciones metropolitanas del mundo, modificaron sustancialmente su base económica, productiva, y social. Una tendencia es la conformación de regiones nucleares (core regions), estos nuevos polos de atracción o aglomeraciones urbanas modifican la dinámica territorial, social y productiva. (Méndez, 2012; Chávez y Guadarrama, 2003)

Las nuevas aglomeraciones metropolitanas se convierten en los motores de la desmaterialización del sistema productivo, debido a la creciente tercerización de la economía, constituida por servicios especializados asociados a la producción y la innovación, que exigen recursos humanos calificados y un ambiente propicio que limita los riesgos de la deslocalización y se convierte en un elemento esencial de las jerarquías interurbanas e intrametropolitanas (Méndez, 2012). En esta perspectiva, el caso mexicano, como cualquier caso de país de industrialización reciente, manifiesta cómo las fuerzas de la globalización se han incorporado al modelo de desarrollo.

En México, la industrialización acelerada se dio con el modelo de Industrialización por Sustitución de Importaciones (ISI) desde los cincuentas hasta inicios de los setenta; sin embargo, la crisis del modelo ISI a mediados de los setenta junto con la crisis del petróleo condujeron a la 'década perdida' en los ochentas. Como respuesta a la crisis, se implementó el modelo de Industrialización Orientado a la Exportaciones (IOE) trayendo consigo políticas de ajuste estructural y apertura comercial implementadas en los noventas, que transformaron la estructura productiva, económica y social, más aún, estos cambios tuvieron una expresión territorial en la Zona Metropolitana de la Ciudad de México (ZMCM) y su región inmediata de influencia: la región del Bajío (Chávez y Guadarrama, 2003).

La visión del desarrollo regional en México, impulsada en el ámbito federal, presupone que las regiones se pueden reinsertar en los mercados globales mediante articulaciones con otros mercados, en la medida que dispongan de ventajas comparativas y competitivas. No se tiene una política explícita ni una estrategia de coordinación de los programas de desarrollo regional. El desarrollo regional y/o sectorial en muchos estados esta desproporcionado, pues la actividad económica está centrada en torno a un área metropolitana cardinal, como son los casos de Aguascalientes, Jalisco, Nuevo León, Puebla, San Luis Potosí y Querétaro. Otras entidades son más policéntricas, es decir, participan varias ciudades, como son los casos de Coahuila (tres), Tamaulipas (cinco), Guanajuato y el Estado de México (seis). (OCDE, 2009; Téllez, 2009).

Empero, existen algunos casos donde pese a la inestabilidad política, derivada de los gobiernos sexenales, se han desarrollado 
opciones innovadoras: Nuevo León, Jalisco y Baja California ${ }^{3}$. Un caso peculiar, donde participan varias entidades que sin tener una coordinación explícita común comparten cultura, conocimiento y tecnologías, es la región conocida como Corredor Industrial de El Bajío $^{4}$ (CIB). El crecimiento económico sostenido de la región del Bajío cimentó su dinamismo en las ventajas locales, que cambió la jerarquía manufacturera de la ZMCM (Chávez y Guadarrama, 2003 y Téllez, 2009).

El objetivo de esta investigación consiste en caracterizar las capacidades del CIB a fin de identificar si este posee cualidades de un ambiente innovador. La metodología empleada consistió en hacer la revisión de literatura disponible; posteriormente, se realizaron una serie de entrevistas semiestructuradas a actores clave que participan en actividades de innovación en la región de estudio. A partir de esta información, se realizó el análisis cualitativo de las características productivas, científicas, tecnológicas y de innovación. El documento se compone de cuatro secciones: la primera es esta introducción, el marco de referencia, continuando con la caracterización de la región de El Bajío que incluye datos generales, análisis de la gobernanza de la región, trayectoria productiva, el desempeño económico y la caracterización como ambiente innovador. Finalmente, se presentan las conclusiones.

\section{Marco de referencia}

La globalización ha provocado el redescubrimiento de la región como una fuerza de desarrollo económico, aunque esto no fue siempre así, como dice O'Riain (2011) estudios recientes muestran que las economías regionales pueden ser constituidas por una variedad de senderos en los que participan

\footnotetext{
3 En Nuevo León, en la ciudad de Monterrey, se viene impulsando el Parque de Innovación e Investigación Tecnológica (PIIT); en Jalisco, en la Zona Metropolitana de Guadalajara se impulsa el Centro de software, y en Baja California, el Border Valley en la Zona Metropolitana de Tijuana.

4 Se entiende por Corredor Industrial del Bajío al conjunto de asentamientos fabriles agrupados en parques o zonas industriales en las colindancias de carreteras con importancia nacional, que va desde San Juan del Río hasta Aguascalientes.
}

una constelación de actores sociales y políticos.

El papel de las regiones se ha retomado con fuerza en la literatura ${ }^{5}$, autores como Allen y Storper (1990) manifiestan que el desarrollo regional se puede analizar como un rompecabezas global con una marcada re aglomeración de la producción y los flujos económicos. Es decir, un mosaico de regiones especializadas con procesos productivos complejos y con creciente interdependencia entre las regiones.

Este trabajo se apoya en el enfoque evolucionista del cambio técnico, al considerar que los elementos territoriales tales como las instituciones y organizaciones son los mecanismos específicos del territorio, como es el path dependence (relaciones sociales, culturales y políticas históricamente construidas) y el proceso de aprendizaje (conocimiento, capacidades, innovación y difusión como elementos que en especificidad al territorio, a nivel de firma o regional o sectorial), así como el papel que juega el mileu innovador, propuesto por el GREMI (donde elementos como la gobernanza, la proximidad, la cooperación y las redes junto con la dinámica y arreglos institucionales le dan coherencia), juegan un papel clave en los territorios, pues ambos enfoques coinciden en que la innovación es un proceso colectivo.

Por ello se afirma que las regiones se han transformado, de huéspedes pasivos que poseen factores y ventajas geográficas, a ser estructuras activas con capacidad de operar proyectos propios que estimulan la formación de sistemas locales donde se promueven y generan procesos de innovación. La identidad de la región se construye como resultado de las estrategias de los actores y del aprendizaje colectivo (Maillat, 1982; Casas y Luna, 2001).

Al mismo tiempo, las empresas innovadoras son atraídas a regiones con recursos e infraestructura de calidad, con sistemas productivos abiertos a la innovación para generar economías externas de escala, así como

\footnotetext{
5 Para una revisión crítica de las diferentes corrientes teóricas véase Fernández et al., 2012.
} 
producir bienes y servicios en condiciones de creciente competencia. Asimismo, estas empresas buscan sitios con una red institucional de apoyo y un clima empresarial favorable a la cooperación y competitividad. Por lo tanto, las regiones ${ }^{6}$ son capaces de definir y ejecutar estrategias de desarrollo que estimulen la dinámica local (Vázquez, 2002).

La región constituye el espacio donde los actores interactúan e intercambian bienes, servicios y conocimientos, con base en reglas específicas. Dado que las regiones han adquirido un renovado papel en la definición de políticas de desarrollo, los gobiernos locales, conscientes de la exposición de sus economías a la competencia global, buscan lograr resultados e impactos reales y positivos al mejorar la competitividad de su economía. (Vázquez, 2002; Casalet y González, 2006).

Otro aspecto a considerar es que las regiones están fundamentalmente ligadas a la economía global a través de mecanismos como el comercio, la exportación y la inversión extranjera y las empresas transnacionales (ETN). Las regiones más competitivas no solo son sedes de empresas locales o del mismo país, sino que resultan atractivas para las firmas más competitivas del mundo. Las regiones se definen cada vez más por los mismos criterios y elementos que una firma intensiva en conocimiento, por lo que deberán adoptar los principios de creación y convertirse en regiones de aprendizaje (Florida, 2000).

Por ello, Vázquez (2002) afirma que la gobernanza o la capacidad de crear acuerdos entre actores y organizaciones, y los resultados en términos de los efectos en la dinámica de las firmas y el desarrollo económico, obedecen a la existencia de un ambiente y un sistema institucional que estimulan los intercambios sociales y políticos y las transacciones económicas. Como dice Cabrero (2005:11), la interacción entre el gobierno y los diversos actores sociales se denomina acción pública. Es por ello que al analizar la

\footnotetext{
6 Se entenderá por región al lugar o espacio en donde se ubican procesos y relaciones sociales y naturales, los cuales, al estar articulados de manera indisoluble, forman un complejo social-natural históricamente determinado (Palacios, 1993).
}

acción pública que deriva en la gobernanza permite encontrar los elementos que dificultan encadenar un desarrollo creciente y sostenido de un territorio.

Un elemento clave en la actividad innovadora a nivel regional es la proximidad, ligada al concepto clásico de "distrito industrial", que ha evolucionado hasta concebirse como la acción de la comunidad que adquiere un dinamismo propio, específico e intrínseco ${ }^{7}$. De acuerdo con Camagni (1991), Vázquez (2002), DiMaggio \& Powell (1983) y Ambos \& Ambos (2009), la proximidad está determinada por cinco ámbitos: a) institucional (reglas y normas que guían la cooperación y coordinación entre actores), b) organizacional (vínculos entre los actores que participan en la producción), c) física (accesibilidad de los factores entre los actores), d) cultural (afinidad cultural de un territorio, facilitando la formación de la identidad) y e) cognitiva (relaciones para el flujo y la transferencia del conocimiento).

De acuerdo con el tipo de la proximidad es posible tener un acceso a las capacidades productivas, tecnológicas, empresariales, de información, incentivos y otras sinergias, las cuales son difíciles de encontrar a distancia, pese a que, aparentemente, las TIC han disminuido la relevancia de la proximidad física o espacial. Lo cierto es que, como afirma Koschatzky (2002), la proximidad espacial por sí misma no resulta suficiente para la cooperación y la formación de las redes, es necesario que coincidan agentes cuyos intereses sean comunes y que estén abiertos a las necesidades de los demás. En ese mismo sentido Gertler (2007) sugiere que la habilidad para producir y compartir conocimiento tácito depende mucho más de la proximidad espacial, afinidad cultural o la cultura corporativa, en particular de la proximidad institucional.

Otro elemento que se asocia a la proximidad es la aglomeración o clústers de empre-

\footnotetext{
7 La literatura de la geografía de la innovación tiene como fundamento las aportaciones de Marshall y posteriormente Perroux entre otros; empero, en los noventa el Groupe de Recherche Européen pour les Milieux Innovateurs (Gremi), colocan nuevamente la geografía económica como tema clave (Pérez, 2011).
} 
sas. Aunque autores como Krugman (1992) dudan de lo novedoso de los clústers basados en el conocimiento, autores como Asheim y Cohen (2005) afirman que la probabilidad de éxito en la actividad innovadora en un clúster tiende a ser mayor en industrias donde el nuevo conocimiento juega un papel importante.

El fenómeno de aglomeración, de acuerdo con Bottazzi et al. (2005), ha tomado diferentes formas, entre las cuales que se encuentran: a) Aglomeraciones horizontalmente diversificadas, caracterizadas por la coexistencia de muchos productores de similares, pero diferentes productos, como los bienes de lujo de "Made in Italy"; b) Formas de aglomeración "smithsonianas", actividades antes integradas verticalmente dentro de firmas individuales que experimentan procesos de división del trabajo y se amplían a diferentes actividades de firmas especializadas, con una cadena de valor local; c) Clústers jerárquicos, en general involucran un núcleo oligopólico, junto con una red de subcontratación, como es la red de proveedores localizados alrededor de las grandes armadoras automotrices; d) Distritos "schumpeterianos", donde la aglomeración es dirigida por las complementariedades en los esfuerzos de innovación, basados en la ciencia, p.e. Silicon Valley y los spin-offs universitarios, los gastos del sector de defensa, el acceso a capital de riesgo y la especialización de las relaciones proveedorusuario y e) Path dependence, con una aglomeración tipo Detroit, fincada en la tradición histórica.

Cada forma de aglomeración, en su momento, representó una forma de innovación social. La velocidad de un proceso innovador depende de la capacidad estratégica de las organizaciones y los vínculos que establecen con los factores contextuales. Los procesos innovadores aparecen como resultantes de la capacidad interna en combinación con las oportunidades del contexto (Cabrero, 2005), de acuerdo con la capacidad estratégica de carácter endógeno que los gobiernos locales y la acción pública local pueden desplegar para incidir sobre el contexto, cada territorio adquirirá diferentes connotaciones ${ }^{8}$.

8 De acuerdo con Elkin y Stone citados por Cabrero (2005) proponen la siguiente tipología de los te-
Un ambiente es innovador si se compone de un sistema de producción, una cultura técnica y sus protagonistas (empresas, asociaciones, autoridades locales, universidades y centros de investigación e individuos) y el proceso de interacción y aprendizaje es llevado a través de un conjunto de redes (formales o no, internas o involucrar externos), y son coherentes, esa es la dimensión cognitiva del mileu, la coherencia (Crevoisier \& Maillat, 1991).

Es por ello que en la búsqueda de opciones de desarrollo local, diversas regiones de Europa, Asia y América Latina, imitando el éxito de otros lugares, implementaron instrumentos como parques científicos, tecnopolos y programas de financiamiento de la innovación, etc. La importancia de la localización de las empresas se convierte en una variable clave para los tomadores de decisiones, al momento de establecer estrategias de desarrollo sustentable y ofrecer incentivos a la IED (Komninos, 2002). También son importantes las políticas y estrategias de desarrollo para la repatriación de los migrantes cualificados (Saxenian, 2007).

Los cambios regionales emanados de los mercados globales, así como el desarrollo de infraestructura para los servicios especializados de las empresas, conforman el nuevo contexto de interacción entre los agentes del sistema de innovación. Son la base del tejido social capaz de coordinar, gestionar e innovar nuevos desarrollos geográficos y sociales. Las nuevas exigencias de calidad en los productos, como el ingreso de IED por los incentivos regionales proporcionados por los gobiernos locales en aras de reciente industrialización, recompone la importancia de las regiones, redefiniendo su vinculación e inserción con el mercado global y nacional, generando nuevas relaciones empresariales y políticas que de acuerdo con las circunstancias recrean nuevas posiciones en el contexto global en el que participa el país (CEPAL, 2000).

rritorios: a) empresarial, dominado por empresas privadas; b) conservador, con aversión a grandes transformaciones, c) progresista, desde los gobiernos locales busca favorecer a las minorías. 


\section{Caracterización de la región El Bajío}

El Bajío posee un amplio potencial de desarrollo económico, al estar ubicado entre dos grandes zonas metropolitanas, la de ciudad de México y la ciudad de Guadalajara, conectadas por importantes vías de comunicación. La región denominada El Bajío, está integrada por algunos municipios de las entidades de Aguascalientes, Guanajuato, Jalisco, Michoacán, Querétaro y San Luis Potosí (Figura $N^{\circ} 1$ ). Debido a su estratégica ubicación geográfica se ha desarrollado un importante corredor industrial.

Figura $N^{0} 1$

Ubicación de las entidades que componen el CIB

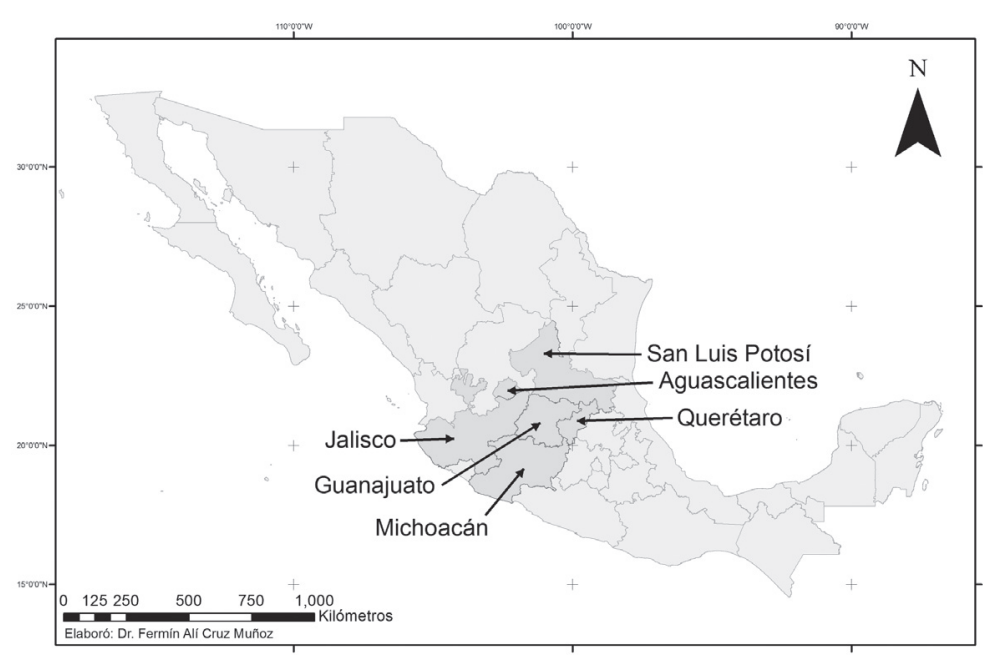

Fuente: Elaboración propia.

Figura $\mathrm{N}^{\circ} 2$

Principales ciudades del CIB

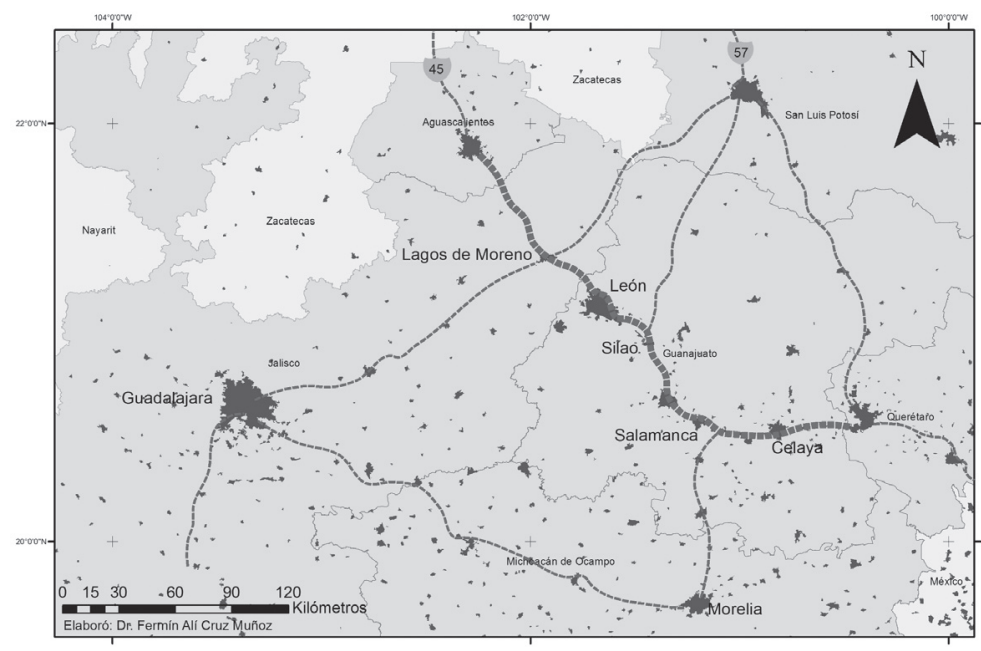

Fuente: Elaboración propia. 
En este corredor industrial se han identificado espacios que ejercen su jerarquía sobre otros sitios inmediatos que les proporcionan cierta independencia funcional del resto del corredor, sin que se trate de espacios conurbanos, como es el caso de las ciudades de Aguascalientes, Querétaro, León, Salamanca e Irapuato. En la región hay una fuerte presencia de ETN y nacionales, asentados en parques industriales donde se está conformando una red territorial productiva, tanto de empresas como de Centros de Investigación, Instituciones de Educación Superior e Institutos Tecnológicos, con lo que se genera una de las mayores economías de aglomeración del país. Como se aprecia en la Figura $N^{\circ} 2$, las ciudades más importantes del CIB están interconectadas por una sólida infraestructura de comunicaciones ${ }^{9}$.

\section{Gobernanza en el corredor}

Detonar el desarrollo regional a través del impulso de una actividad clave de la economía o bien para alcanzar la convergencia de las diferentes regiones. Estos intentos en México, se han caracterizado por contener, invariablemente los mejores propósitos pero en su ejecución y, sobre todo, en la consecución de resultados han mostrado su vulnerabilidad, entre otras cosas, cuando son relegados por parte de los tomadores de decisiones (García, 2008).

Como ya se mencionó, en México no existen políticas públicas regionales explícitas, lo que hay son políticas estatales, en buena medida esta situación está asociada a los insuficientes avances en la descentralización de presupuesto público federal para la infraestructura social.

El caso del Bajío es sui generis por dos elementos que se retroalimentan mutuamente: el primero, la continuidad en los proyectos de desarrollo, pese a que en la región coexisten la alternancia de partidos (Aguascalientes, Jalisco, Michoacán, Querétaro y

\footnotetext{
9 La función territorial del transporte permite analizar la concentración y dispersión de la infraestructura, y los procesos de concentración de la población y de la actividad económica territorial (Unger et al., 2013)
}

San Luis Potosí) en el poder estatal y municipal y la permanencia política (Guanajuato). El segundo, el proceso de restructuración productiva de la región, que aprovecha la restructuración productiva a nivel mundial de la inversión extranjera directa (IED), la ubicación estratégica y las ventajas del Tratado de Libre Comercio de América del Norte (TLCAN).

La continuidad de los proyectos de desarrollo se debió a la construcción de la gobernanza y la acción pública, que pese a la escasa descentralización en la región dio cabida a un proceso de gobernanza a nivel estatal y que creó un proceso de isomorfismo ${ }^{10}$ institucional y de políticas de desarrollo de las entidades vecinas. Particular importancia tiene el Sistema de Planeación Estatal, pues ha sido un factor importante que ha ayudado a restar importancia a la centralización que privó durante el modelo Industrialización por Sustitución de Importaciones en México y le dio estabilidad al desarrollo local con independencia de los gobiernos en turno (Téllez, 2009). Aunque no todas las entidades mostraron un desarrollo similar, se observa que las entidades donde está ubicado el CIB sí sufrieron un proceso isomórfico en su gobernanza.

La gobernanza de la región se puede analizar desde dos perspectivas: el desarrollo institucional a nivel estatal y las iniciativas colectivas. En el primer caso, el desarrollo institucional estatal está ligado al proceso iniciado a nivel federal durante los años setenta con la creación de instancias de promoción, y después de coordinación y planeación en todos los estados del país ${ }^{11}$. Aunque todas las entidades de El Bajío siguieron las pautas marcadas desde la federación, su contexto y propia trayectoria fueron dando cabida a dife-

\footnotetext{
10 Isomorfismo es el proceso que impulsa a una población a parecerse a otras unidades de la misma área, en el mismo conjunto de condiciones ambientales. Es decir, cuando un ambiente presiona con fuerza a las organizaciones a operar con mejores prácticas, ser eficaces y eficientes (DiMaggio y Powell, 1983).

11 En los setenta se conformaron los Comités Promotores del Desarrollo del Estado (Coprode) en el país, en 1981 se trasforman en Consejos de Planeación para el Desarrollo Estatal (Coplade), para coordinar los tres niveles de gobierno y ser el canal entre el sistema de Planeación Nacional y Estatal. (Narváez, s/f).
} 
Cuadro $\mathrm{N}^{\circ} 1$

Desarrollo institucional de las entidades de El Bajío

\begin{tabular}{|c|c|c|}
\hline Estado & $\begin{array}{c}\text { Naturaleza del desarrollo institucional } \\
\text { estatal }\end{array}$ & Mecanismos de planeación estatal \\
\hline 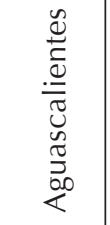 & $\begin{array}{l}\text { A fines de los ochenta se creó la Secretaría } \\
\text { de Planeación y Desarrollo, después cambió } \\
\text { a Administración del Desarrollo, a mediados } \\
\text { de los noventa se incorporó una parte a de- } \\
\text { sarrollo urbano y desarrollo social. }\end{array}$ & $\begin{array}{l}\text { Comité de Planeación para el Desarrollo } \\
\text { del Estado (Coplade) y los subcomités sec- } \\
\text { toriales. }\end{array}$ \\
\hline 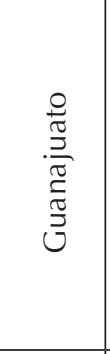 & $\begin{array}{l}\text { Inicia en los noventa, cuando la ciudad de } \\
\text { León es pivote de la evolución institucional. } \\
\text { Otros municipios no han desarrollado ini- } \\
\text { ciativas propias sino que desde el gobierno } \\
\text { estatal se ha implantado acciones desde el } \\
\text { Copladeg. } \\
\text { El Copladeg instala en cada región un con- } \\
\text { sejo regional y las presenta ante el ejecutivo } \\
\text { estatal. }\end{array}$ & $\begin{array}{l}\text { El Consejo de Planeación para el Desarro- } \\
\text { Ilo del Estado de Guanajuato (Copladeg) } \\
\text { coordina al Consejo Sectorial de Desarrollo } \\
\text { Económico y Medio Ambiente, al Consejo } \\
\text { Sectorial de Educación, Cultura y Recrea- } \\
\text { ción, a los representantes de la Secretaría } \\
\text { de Desarrollo Económico Sustentable y a } \\
\text { los representantes de los ayuntamientos de } \\
\text { las seis regiones del estado. }\end{array}$ \\
\hline 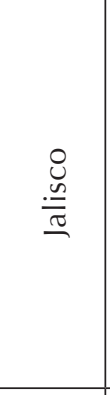 & $\begin{array}{l}\text { El Consejo de la Zona Metropolitana de } \\
\text { Guadalajara (ZMG), en colaboración con } \\
\text { el gobierno municipal, los organismos gre- } \\
\text { miales y empresariales buscó regularizar el } \\
\text { crecimiento urbano, operó desde los treinta } \\
\text { y dejó de operar en los setenta. } \\
\text { El Consejo Estatal de Promoción Económica } \\
\text { (CEPE, creado en 1997) promueve la inver- } \\
\text { sión. }\end{array}$ & $\begin{array}{l}\text { El Consejo ZMG coordina y concertar la } \\
\text { gestión de las acciones intermunicipales. } \\
\text { Programas para el desarrollo regional: el } \\
\text { Fondo para el Desarrollo Regional (Fonde- } \\
\text { reg), Fondo Metropolitano, etc. } \\
\text { El Copladereg y el Fondereg fomentan la } \\
\text { pertenencia regional y sustentablilidad de } \\
\text { los recursos. } \\
\text { Ley de Fomento Económico y los incentivos } \\
\text { fiscales para la atracción de inversiones. }\end{array}$ \\
\hline 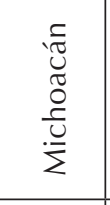 & $\begin{array}{l}\text { Las iniciativas para la planeación de la } \\
\text { expansión urbana de Morelia no han ad- } \\
\text { quirido la relevancia necesaria, pese al } \\
\text { reconocimiento de la preservación de la } \\
\text { biodiversidad de la entidad. }\end{array}$ & $\begin{array}{l}\text { Comité de Planeación para el Desarrollo } \\
\text { del Estado de Michoacán (Copladem) y la } \\
\text { operación de diez Subcomités Sectoriales. }\end{array}$ \\
\hline 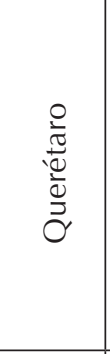 & $\begin{array}{l}\text { El programa Estatal de Fomento Industrial } \\
\text { 1987-1991 impulsó la instalación de empre- } \\
\text { sas en el Corredor Industrial Querétano. } \\
\text { A fines de los noventa, el fideicomiso In- } \\
\text { dustrial del Estado de Querétaro (Fideqro) } \\
\text { impulsó el corredor urbano ecológico } \\
\text { industrial de San Juan del Río-Querétaro, } \\
\text { como eje ordenador a la autopista México- } \\
\text { Querétaro. }\end{array}$ & $\begin{array}{l}\text { El Comité de Planeación para el Desarrollo } \\
\text { del Estado de Querétaro (Copladeq) y los } \\
\text { subcomités. } \\
\text { El Instituto Municipal de Planeación de la } \\
\text { ciudad de Querétaro (Impla) en 2004. } \\
\text { Comité Promotor del Desarrollo Económi- } \\
\text { co del Estado de Querétaro y la Promotora } \\
\text { de Empresas del Estado de Querétaro. }\end{array}$ \\
\hline 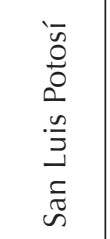 & $\begin{array}{l}\text { El Sistema de planeación urbana de la ciu- } \\
\text { dad de San Luis Potosí tomó suficiente auto- } \\
\text { nomía para proponer un desarrollo integral } \\
\text { (1995). }\end{array}$ & $\begin{array}{l}\text { Comité de Planeación del Desarrollo Es- } \\
\text { tatal, Instituto Municipal de Planeación } \\
\text { y después Instituto de Planeación para el } \\
\text { Desarrollo Sustentable de San Luis Potosí. }\end{array}$ \\
\hline
\end{tabular}

Fuente: Elaboración propia en base a Téllez (2009); Cabrero (2005); Sainz (s/f); González, G. (2005); González, S. (2005). 
rentes alternativas de planeación, en términos de la duración, participación social e intensidad de las acciones realizadas, por lo que es un elemento de desarrollo endógeno.

En el Cuadro $\mathrm{N}^{\circ} 1$ se sintetiza el desarrollo institucional reciente de cada entidad de la región. Se observa que no todas las entidades tuvieron un desarrollo equiparable, aunque han adoptado políticas similares de atracción de IED y cuyo eje aglutinador fue una metrópoli, con excepción de Michoacán que ha quedado rezagado. En el caso de Querétaro, Aguascalientes y San Luis Potosí, adoptaron procesos similares a los que encabezaron Jalisco ${ }^{12}$ y Guanajuato.

En el ámbito regional, en 1999 iniciaron los esfuerzos para conformar la Región Centro-Occidente (que comprende los estados de Aguascalientes, Colima, Guanajuato, Jalisco, Michoacán, Nayarit, Querétaro, San Luis Potosí y Zacatecas), se buscó "construir una visión regional compartida por los nueve estados y definir una estrategia integral para el desarrollo de la región". Se conformó el Sistema de Información de la Región Centro Occidente (Sirco), se realizaron reuniones periódicas entre dependencias homólogas, en cuatro vertientes: económica, social, ambiental y territorial (ordenamiento e infraestructura).

Desde entonces, el proceso regional se ha venido consolidando. En diciembre de 2001 se constituyó el Fideicomiso para el Desarrollo de la Región Centro-Occidente

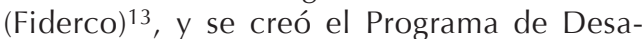
rrollo de la Región Centro Occidente ${ }^{14}$ como los instrumentos para lograr la operación del modelo de planeación regional.

12 Guadalajara en los años cuarenta del siglo XX, creo una vicepresidencia municipal, que ocupaban empresarios (Alva et al., 1998), un caso único en el país.

13 Se integró con aportaciones de los estados, del Gobierno Federal y donaciones de terceros, dichos recursos fueron destinados para apoyar acciones, estudios y proyectos regionales.

14 Primer instrumento a nivel nacional, de carácter programático, a mediano y largo plazo para el desarrollo integral de la región. http://www.centroccidente.org. $\mathrm{mx} /$
Sin embargo, como señala Téllez (2009), los planes de desarrollo regional tienen lugar sin que necesariamente coexista la suficiente intencionalidad política que garantice su cumplimiento, por lo que los alcances apenas son perceptibles, pero la constante interacción y las necesidades comunes han ido acercando a los actores de la región, derivado en buena medida de las sinergias de las propias trayectorias tecnoproductivas de cada entidad. La interacción es implícita, pero no se identificaron acciones o programas que dieran continuidad a esta forma de gobernanza.

Sin embargo, como señala Téllez (2009), los planes de desarrollo regional tienen lugar sin que necesariamente coexista la suficiente intencionalidad política que garantice su cumplimiento, por lo que los alcances apenas son perceptibles, pero la constante interacción y las necesidades comunes han ido acercando a los actores de la región, derivado en buena medida de las sinergias de las propias trayectorias tecnoproductivas de cada entidad. La interacción es implícita, pero no se identificaron acciones o programas que dieran continuidad a esta forma de gobernanza.

\section{Trayectoria productiva del CIB}

La trayectoria productiva de las seis entidades que componen el CIB ha transitado por tres vías: una basada en la explotación de los recursos disponibles (agrícola y minería), otra fundada en la manufactura (química, alimentos, calzado y manufacturas diversas) y la tercera, que incorpora nuevas tecnologías (software, automotriz y aeronáutica). A continuación se describe en forma sucinta la trayectoria productiva de las entidades que conforman el CIB.

\section{Aguascalientes}

El proceso de industrialización de Aguascalientes, de acuerdo con Rojas et al. (1994) y Camacho (2005), tiene tres etapas:

a) Tejido industrial embrionario: hasta los ochenta predominó la producción de alimentos, metalmecánica, textil y de la confección. En los sesenta adquirieron relevancia los talleres y fábricas de alimentos, textil, confección y metalmecánica, 
además de centro comercial y nodo ferroviario a escala nacional; a fines de esa década el sector textil y confección atravesaba por una fuerte crisis. Esta situación creó la necesidad de impulsar un nuevo patrón de crecimiento económico.

b) Atracción de inversión extranjera y el desarrollo del clúster automotriz: a partir de los ochenta, se convirtió en un importante receptor de IED en las ramas de metalmecánica y electrónica, cuya producción se orientaría al mercado externo. Esta situación derivó en que las pymes textil y de confección fueran relegadas. En la rama metalmecánica, aunque los talleres ferroviarios entraron en decadencia, empresas de equipos metálicos se transformaron y se convirtieron en líderes nacionales. La industria de la construcción, debido a la urbanización y obra pública, estuvo en auge. Con la instalación de la planta de Nissan (1981) inició el complejo automotriz que aún sigue creciendo con empresas de autopartes nacionales e internacionales. En ese mismo periodo se asentó Xerox (1983) y Texas Instrument (1984) creando una red de proveedores. En los noventa, el proceso de globalización prosiguió extendiéndose con la instalación de maquiladoras, en el sector de ensamble de prendas de vestir y metalmecánica, este tipo de aglomeración correspondería a los clústers jerárquicos.

\section{Guanajuato}

La industrialización de Guanajuato se presentó en tres direcciones, de acuerdo con Blanco et al. (2000), Téllez (2009) y Martínez et al. (2009):

a) Agricultura comercial a gran escala: ligada a la creación del distrito de riego del Alto Lerma en 1933. El agro guanajuatense presenta una tendencia hacia el aumento en la producción de cultivos comerciales en detrimento de los productos básicos. El sorgo, trigo y cebada ocuparon la mayor parte de la superficie cultivada, sin embargo, la contribución al PIB estatal de las hortalizas es mayor, resultado de una agricultura intensiva. Las principales procesadoras de hortalizas y agroindustrias en el estado son ETN. En este caso este tipo de aglomeración correspondería a "clústers jerárquicos".

b) Subsistencia del tejido industrial local: Las empresas de calzado y curtido de piel han logrado incrementar su presencia al colocar al estado en el lugar número once como exportador a escala nacional. La consolidación de ramas ya establecidas en la región, donde la gran mayoría de las empresas son pequeños talleres o microempresas y solo $5 \%$ de ellas han logrado incursionar en el mercado internacional. Esta sería una "aglomeración horizontalmente diversificada".

c) Enclaves energéticos y atracción de inversión extranjera: la inauguración de la refinería de Pemex en Salamanca (1950) y la planta termoeléctrica en el mismo municipio (1968), permitió que la actividad de la industria química y petroquímica se concentrara en torno a la refinería. La instalación de General Motors (GM) en Silao (1994) y la operación del Puerto Interior (2006) aceleró la atracción de industrias de equipo de transporte, petroquímica, metalmecánica, textil, eléctrica, alimentaria y agroindustria. En cambio, la industria automotriz y de autopartes se aglomera alrededor de la planta de GM. Este tipo de aglomeración sería a "smithsoniana", pues hay una cadena de valor local.

\section{Querétaro}

El proceso de industrialización del estado. De acuerdo con García (1999) y Pérez (2014) este tiene tres dimensiones:

a) Creación de parques industriales: en los sesenta se formó la Unidad de Parques Industriales. En los ochenta, se instalaron empresas terminales en estados cercanos (Chrysler en el estado de México, Nissan en Aguascalientes, Dina en Hidalgo y GM en Guanajuato), colocando al estado en una posición estratégica en términos de acceso a mercados (nacionales e internacionales), acceso logístico y de comunicaciones y de ambiente privilegiado (estímulos fiscales y disponibilidad de mano de obra). Se dio preferencia al desarrollo industrial intensivo en capital, centrado en la industria de autopartes, fabricación 
de piezas, maquinaria y equipo. Es decir, se presenta como parte de la "aglomeración jerárquica" en torno a la industria automotriz de las entidades próximas.

b) Especialización productiva: la estructura diversificada de la manufactura, con algunas industrias desarrolladas, como equipo de transporte (ensamble de automóviles y autopartes); alimentos, bebidas y tabaco; productos químicos, plásticos, hule y minerales no metálicos; y papel e impresión. En la última década se han desarrollado los sectores de alta tecnología, como es la industria aeroespacial que giran alrededor de General Electric y Bombardier y el desarrollo del clúster aeroespacial denominado Parque Industrial Aeroespacial. El path dependece que Querétaro ha desarrollado en metalmecánica como elemento que posibilita la incorporación a la industria aeronáutica.

\section{Jalisco}

El proceso de industrialización jalisciense tuvo dos etapas, de acuerdo con González S. (2005):

a) Tejido industrial local: predominado por las pyme de productos tradicionales como alimentos, calzado, textiles, artesanías y otros, que cubrían las necesidades del mercado interno y de la región de occidente. La industria local no se había modernizado y no estaba preparada para competir en el mercado internacional. El gran comercio pasó a mano de los capitales extranjeros y algunos se aliaron con al comercio local. Los pequeños empresarios desplazados por el calzado, ropa y juguetes del sureste asiático, desaparecieron o son distribuidores.

b) Atracción de la industria moderna: a partir de los años sesenta, impulsada por el gobierno estatal con incentivos económicos y disponibilidad de parques industriales, así como mano de obra calificada. Las empresas más importantes que llegaron a la región fueron Kodak (1985), Burroughs, Motorola (1968), IBM (1975), Hewlett-Packard (1982) y Lucent technologies (1990), buscaban aprovechar el programa de importación temporal y más tarde se acogieron al régimen de maquiladora temporal. Estas empresas sirvieron de atracción a otras, proveedoras de insumos y materiales. Lo que permitió la integración horizontal lo que sería el antecedente del surgimiento de la industria electrónica. Entre 1995 y 2000 Ilegaron una cantidad importante de IED, 47 empresas de las 70 que se tienen registradas en la rama electrónica, que generan $28 \mathrm{mil}$ empleos directos y más de 100 mil empleos indirectos. La mayoría del comercio es hacia EE.UU., es decir, comercio intrafirma. Una aglomeración de tipo jerárquico.

\section{Michoacán}

El desarrollo industrial ha estado asociado con la disponibilidad de materias primas. De acuerdo con Ochoa y Sánchez (2003) y Muría (1994) está caracterizado por tres momentos:

a) Tejido industrial local diverso: durante los cuarenta las pequeñas factorías, poco mecanizadas, de alimentos y una relativa explotación de recursos forestales y en menor medida de textil. La posguerra trajo consigo el auge de la industria textil e industrias diversas, tales como una empresa de equipo de medición, despepitadoras, insecticidas, aceite de limón, muebles, accesorios de madera, láminas de cartón y empacadoras de frutas y legumbres, producción y procesamiento de carne porcícola.

b) Instalación de industrial integradoras: en los cuarenta se estableció Celanese Mexicana, dedicada a la fabricación de fibras sintéticas y con ella otras empresas químicas como la industria extractiva asociada a la Siderúrgica Lázaro Cárdenas, explotación de yacimientos de minerales metálicos y no metálicos. La instalación de plantas de fertilizantes (distribuidores de Pemex) y otras industrias dinamizaron la región del puerto. En los setenta se construyó el parque industrial Morelia, con Industrias Resistol y turbinas, creando obra pública e incentivos para grandes empresas. Este modelo corresponde a las "formas de aglomeración smithsonianas", por su integración vertical. 
c) Especialización agrícola y forestal: las obras de riego impulsaron el cultivo de caña de azúcar, limón, aguacate y fresa. La explotación forestal también alcanzó niveles considerables, la Unión Forestal de Jalisco y Colima fue la más importante. Posteriormente se estableció la fábrica de papel en 1946 que hasta los setenta tenía una producción importante. También creció la industria del tequila aunque solo para consumo nacional.

\section{San Luis Potosí}

La estructura industrial del estado, de acuerdo con Pastor y Medina (2008), OCDE (2009) y Moreno et al. (2008), se compone de dos dimensiones:

a) Industria local: la industria metalúrgica básica tiene la mayor participación en la generación de riqueza. Los principales productos son zinc, cobre, plomo, oro, plata, mercurio, plomo, manganeso y arsénico. Otra industria es la química, también hay empresas pymes de textiles, alimentos y bebidas.

b) Polo de manufactura: la industria automotriz y de autopartes es un sector clave de la estrategia de modernización e integración de San Luis Potosí en la economía mundial. El grado de especialización en la rama de autopartes del estado, así como la dinámica del sector, se constituye como un clúster con amplias ventajas en los ámbitos regional y nacional, inicialmente en autopartes y en la actualidad en la industria terminal de automóviles. Esto le permite promover la formación del clúster automotriz, pues cuenta con diez parques y zonas industriales. Se está en presencia de clústers jerárquicos.

\section{Desempeño del CIB}

La vocación productiva de la región se ha enfocado a los sectores automotriz, autopartes, electrónica y alimentos. La atracción de capital en la región del Bajío entre 1989 y 2013 reportó 2.305,1mdd, generando cerca de 100 mil empleos directos (Téllez, 2009 y Secretaría de Economía, 2014).
Al analizar el desempeño de las entidades que componen la región, en los casos de Jalisco y Guanajuato emprendieron opciones de desarrollo propias, Guanajuato optó por la diversificación (alimentos, metalmecánica y calzado) y Jalisco por explotar las ventajas de aglomeración (electrónica y metalmecánica).

En los casos de Aguascalientes, San Luis Potosí y Querétaro, se observa un proceso de convergencia productiva orientada a la industria metalmecánica y un isomorfismo institucional que no ha culminado. Michoacán no ha seguido ese sendero, pero tampoco se vislumbra que esté desarrollando una estrategia propia. Por ello, como señala Téllez (2009), la región más homogénea, puesto que Michoacán no se ha integrado al corredor, Aguascalientes, Guanajuato, Jalisco, Querétaro y San Luis Potosí, debido a que presentan una convergencia productiva.

Cabe mencionar que en la región del CIB existe una cultura emprendedora, no solo las políticas públicas incentivan la creación de nuevas empresas, sino que también los apoyos del sistema financiero $y$, en términos sociales, ser empresario se considera un logro individual y social, que facilita los procesos de Transferencia de Tecnología (Pérez, 2014).

Si se definiera un estado con más éxito, de acuerdo a la información económica del Cuadro $\mathrm{N}^{\circ} 2$, sería Jalisco, pero si consideramos los elementos de desarrollo Querétaro y Aguascalientes son quienes tienen mejor desempeño a nivel social y económico.

Aunque autores como OCDE (2009), Unger et al. (2013) y Pérez (2014) afirman que al analizar la estructura productiva, relacionada con los sectores más dinámicos del crecimiento económico de la región del Bajío, se observa que no está suficientemente integrada en cadenas de valor que generen sinergias regionales y la incorporación de conocimiento local a esas cadenas es incipiente.

\section{Elementos del corredor como región innovadora}

EI CIB se fue creando a partir de la proximidad física, sin una intencionalidad clara, pero por la necesidad y competencia de 


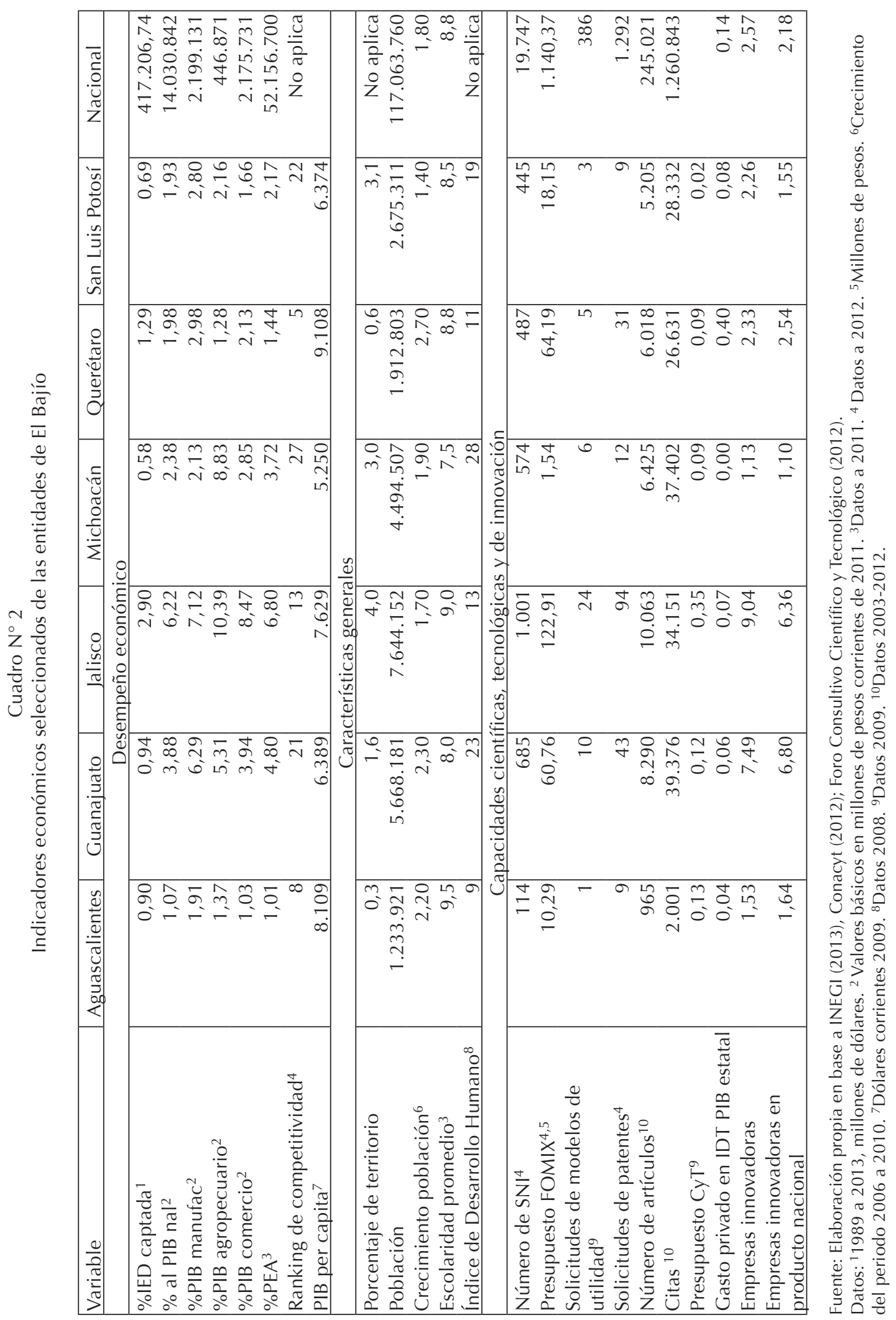


atraer mayores inversiones a sus respectivos estados, asimismo, desarrolló un proceso de gobernanza local, lo que le abrió ventanas de oportunidad, no obstante que las estrategias para fomentar la innovación en los estados son distintas, como apunta Pérez (2014).

La trayectoria del CIB incluye una región creada a partir de desarrollos propios de cada ciudad, como la industria del calzado y textil Path dependence. Adicionalmente, se tiene presencia de clústers jerárquicos en casos como la petroquímica, agroindustria y autopartes. Ambas formas de concentración conviven y se renuevan constantemente. Por tanto, en donde la proximidad física fue un elemento inicial, conjugado con el desarrollo institucional, fue generando las condiciones mínimas para el desarrollo local.

Lo anterior sentó las bases que derivaron en la atracción de inversiones y nuevos giros económicos que han transformado para siempre la región de El Bajío, de ser 'el granero de México' al 'corazón fabril de México'.

Es importante señalar que el CIB comparte la cultura del Bajío, lo que facilitó no solo la movilidad sino también compartir prácticas y conocimientos. Si bien, como se analiza en el Cuadro $N^{\circ} 3$, la explotación del conocimiento es un área de oportunidad, lo es también el ambiente industrial que está comenzado a dar los pasos para transformarse en un ambiente innovador pese a tener una débil organización conjunta, por lo que la coherencia está aún en proceso de construcción.

A partir de la búsqueda de opciones de desarrollo y basada en disponibilidad de mano de obra y accesibilidad de la infraestructura, permite la movilidad entre los diferentes actores del CIB e incentivos para la atracción de IED, se aprovechan las ventajas de localización en la región para atraer empresas de mayor complejidad tecnológica.

Como se observa en la Cuadro $N^{\circ} 3$, el CIB tiene elementos que apuntan a afirmar que se está en presencia de un ambiente innovador en construcción, aunque la coordinación (o proximidad institucional) entre las diferentes entidades es un elemento clave que deberá robustecerse para lograr un mayor avance futuro. Se observan cinco diferentes

Cuadro $\mathrm{N}^{\circ} 3$

Características del ambiente innovador del CIB

\begin{tabular}{|c|c|c|c|}
\hline Estado & $\begin{array}{c}\text { Tipo de proximidad } \\
\text { detectada }\end{array}$ & Aprendizaje identificado & Redes presentes \\
\hline 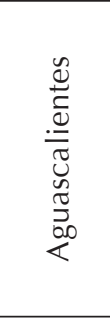 & $\begin{array}{l}\text { Economías de aglome- } \\
\text { ración alrededor de los } \\
\text { parques industriales }\end{array}$ & $\begin{array}{l}\text { Creación de derramas a partir } \\
\text { de la IED, para crear la cade- } \\
\text { na de proveedores y parques } \\
\text { de alta tecnología aunque con } \\
\text { éxito limitado. } \\
\text { Ramas dinámicas: autopartes, } \\
\text { transporte, eléctrico/ electró- } \\
\text { nico y TIC. }\end{array}$ & $\begin{array}{l}\text { Formación de capital huma- } \\
\text { no para aumentar el nivel de } \\
\text { cualificación del empleo que } \\
\text { requieren las ETN. } \\
\text { Busca desarrollar cadena de } \\
\text { proveedores de mayor comple- } \\
\text { jidad tecnológica. }\end{array}$ \\
\hline 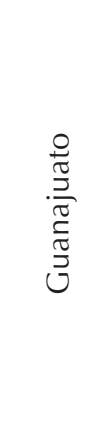 & $\begin{array}{l}\text { Actividad de CyT inten- } \\
\text { siva y diversificada en } \\
\text { el CIB. } \\
\text { No se han desarrollado } \\
\text { suficientes mecanismos } \\
\text { de traducción para lo- } \\
\text { grar la explotación del } \\
\text { conocimiento de fron- } \\
\text { tera que se genera en la } \\
\text { entidad, existen casos } \\
\text { aislados de éxito. }\end{array}$ & $\begin{array}{l}\text { Política pública local de apo- } \\
\text { yo a la innovación. } \\
\text { Esfuerzos para fortalecer las } \\
\text { capacidades tecnológicas de } \\
\text { las pymes,. Busca difundir las } \\
\text { mejores prácticas entre las } \\
\text { pymes. } \\
\text { Áreas más dinámicas: auto- } \\
\text { motriz, autopartes, metalme- } \\
\text { cánica, cuero y calzado. }\end{array}$ & $\begin{array}{l}\text { Fuerte relación entre la indus- } \\
\text { tria y el Concyteg y otras enti- } \\
\text { dades públicas, y participación } \\
\text { alta en casi todos los progra- } \\
\text { mas del Conacyt. } \\
\text { Dispone de una gran cantidad } \\
\text { y gama de actores, en diferen- } \\
\text { tes niveles de complejidad y } \\
\text { con distintos niveles de traduc- } \\
\text { ción de las necesidades de las } \\
\text { empresas. }\end{array}$ \\
\hline
\end{tabular}




\begin{tabular}{|c|c|c|c|}
\hline Estado & $\begin{array}{c}\text { Tipo de proximidad } \\
\text { detectada }\end{array}$ & Aprendizaje identificado & Redes presentes \\
\hline 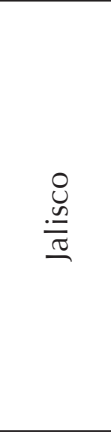 & $\begin{array}{l}\text { Economías de aglome- } \\
\text { ración alrededor de los } \\
\text { parques industriales de } \\
\text { la ZMG. } \\
\text { Alrededor de los par- } \\
\text { ques industriales se han } \\
\text { localizado las incubado- } \\
\text { ras de empresas. }\end{array}$ & $\begin{array}{l}\text { Disponen de una gran can- } \\
\text { tidad de instrumentos para } \\
\text { la promoción, divulgación y } \\
\text { vinculación de CyT. } \\
\text { Centra sus esfuerzos en la for- } \\
\text { mación de personal calificado } \\
\text { para las necesidades de las } \\
\text { empresas en la región. } \\
\text { Áreas de mayor dinamismo: } \\
\text { agroalimentaria, TIC, anima- } \\
\text { ción y microelectrónica. } \\
\end{array}$ & $\begin{array}{l}\text { Se han impulsado sectores } \\
\text { como automotriz y TIC, empe- } \\
\text { ro no han tenido mucho éxito. } \\
\text { Desarrollaron una convocato- } \\
\text { ria espejo propia de estímulos } \\
\text { fiscales a la innovación y un } \\
\text { programa específico para la } \\
\text { promoción de la vinculación } \\
\text { academia-empresa. }\end{array}$ \\
\hline 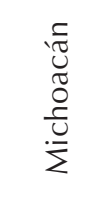 & $\begin{array}{l}\text { Diversificación de la } \\
\text { economía a lo largo del } \\
\text { territorio ha creado islas } \\
\text { de desarrollo industrial. }\end{array}$ & $\begin{array}{l}\text { temas de } \\
\text { vación sin } \\
\text { ctor. } \\
\text { dinamismo }\end{array}$ & $\begin{array}{l}\text { Hay retos en la coordinación } \\
\text { institucional para la operación } \\
\text { de los programas de CyT, se- } \\
\text { guido del tema de infraestruc- } \\
\text { tura. }\end{array}$ \\
\hline 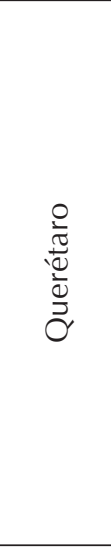 & $\begin{array}{l}\text { Busca fomentar las ca- } \\
\text { pacidades de ciencia y } \\
\text { tecnología, y ausencia a } \\
\text { la promoción de la acti- } \\
\text { vidad de innovación. } \\
\text { Dispone del mayor nú- } \\
\text { mero de centros de in- } \\
\text { vestigación después del } \\
\text { DF. } \\
\text { Resistencia de las pymes } \\
\text { a la colaboración, salvo } \\
\text { aquellas que han emer- } \\
\text { gido de incubadoras de } \\
\text { empresas. }\end{array}$ & $\begin{array}{l}\text { Experiencia industrial en ali- } \\
\text { mentos, metalmecánica y au- } \\
\text { topartes. } \\
\text { Vínculos aislados de empresas } \\
\text { grandes con organizaciones } \\
\text { de apoyo a la innovación. } \\
\text { Se está atrayendo a la indus- } \\
\text { tria aeronáutica basada en las } \\
\text { capacidades generadas en la } \\
\text { metalmecánica y autopartes. } \\
\text { Ramas dinámicas tecnológi- } \\
\text { camente son aeronáutica, TIC, } \\
\text { nuevos materiales, automotriz } \\
\text { y electrodomésticos. }\end{array}$ & $\begin{array}{l}\text { Fuerte interacción con las em- } \\
\text { presas asentadas en el DF. } \\
\text { Se han desarrollado mecanis- } \\
\text { mos alternos para coadyuvar a } \\
\text { la cooperación entre organiza- } \\
\text { ciones empresariales y la aca- } \\
\text { demia con impacto limitado. } \\
\text { Escasa colaboración empresas- } \\
\text { academia para proyectos de } \\
\text { innovación. } \\
\text { Financiamiento de la inno- } \\
\text { vación con fondos federales } \\
\text { y centrados en el desarrollo } \\
\text { tecnológico. }\end{array}$ \\
\hline $\begin{array}{l}\bar{n} \\
0 \\
0 \\
0 \\
. n \\
\Xi \\
\bar{J} \\
\tilde{\sigma}\end{array}$ & $\begin{array}{l}\text { Las industrias maquila- } \\
\text { dora y manufactura de } \\
\text { autopartes aprovechan } \\
\text { la proximidad con otras } \\
\text { entidades de El Bajío. } \\
\text { Se brindan servicios tec- } \\
\text { nológicos localmente de } \\
\text { bajo nivel, son pocos los } \\
\text { oferentes y centrados en } \\
\text { las industrias más ren- } \\
\text { tables. }\end{array}$ & $\begin{array}{l}\text { Las organizaciones interme- } \\
\text { dias funcionan como gestoras } \\
\text { de recursos públicos y para } \\
\text { la difusión de conocimiento, } \\
\text { por lo que hay escasez en la } \\
\text { función de traducción. } \\
\text { Derramas escasas de cono- } \\
\text { cimiento de las otras filiales } \\
\text { dentro del ClB. } \\
\text { Las ramas más dinámicas son: } \\
\text { automotriz y autopartes, ae- } \\
\text { roespacial, metalmecánico, } \\
\text { acero y plástico. }\end{array}$ & $\begin{array}{l}\text { Participación en los fondos } \\
\text { nacionales relacionados con } \\
\text { la innovación es menor, a di- } \\
\text { ferencia de los concernientes a } \\
\text { aspectos científicos. La recien- } \\
\text { te participación en redes de in- } \\
\text { novación alrededor del Ipicyt } \\
\text { es creciente; con el apoyo del } \\
\text { Copocyt, brinda el respaldo } \\
\text { necesario para el otorgamiento } \\
\text { de recursos públicos de impul- } \\
\text { so a la innovación. }\end{array}$ \\
\hline
\end{tabular}

Fuente: Elaboración propia con base en entrevistas Plan Estatal de Ciencia y Tecnología de Aguascalientes 2009-2020; Programa Estatal de Ciencia y Tecnología de Michoacán 2008-2012; Programa Estatal de Ciencia, Tecnología e Innovación de Querétaro 2010-2015. Programa Estatal de Ciencia y Tecnología de Guanajuato 2008-2012. Plan Estatal de Desarrollo 2009-2015 de San Luis Potosí. Programa Sectorial de Ciencia y Tecnología para el Desarrollo de Jalisco. 
tipos de proximidad (institucional, organizacional, física, cultural y cognitiva), en el caso de la cognitiva hay escasa evidencia del aprendizaje colectivo y redes de colaboración, en buena medida derivado de que en el CIB comparten trayectorias productivas comunes, con necesidades y cultura compartida.

Finalmente, los elementos que definen al CIB como ambiente son: dispone de infraestructura de calidad, aunque en forma poco coordinada, tiene sistemas productivos abiertos a la innovación y, debido a la presencia de economías de aglomeración, se generan también economías externas a escala. Además se producen productos y servicios en condiciones de creciente competencia. La constante atracción de IED y nuevas industrias dan evidencia también de la red de apoyo institucional, por lo que está respaldado por un clima propicio para la cooperación y la competitividad. Por lo anterior se puede afirmar que el CIB es un ambiente innovador pues define y realiza estrategias de desarrollo que estimulan la dinámica local. Que, aunque no se trata de una estrategia colectiva, las entidades de la región imitan el comportamiento de las entidades vecinas o lo que aquí se denomina "isomorfismo regional".

\section{Reflexiones finales}

El papel de las regiones ha cambiado. La paradoja donde la actividad innovadora no está distribuida de manera uniforme en el espacio geográfico y la tendencia hacia la concentración espacial a través del tiempo, distinguen territorios que reconvirtieron sus ventajas comparativas en ventajas competitivas con el fin de atraer un tipo distinto de inversión que no solo busca exenciones fiscales en la mano de obra sino que también busca un entorno que favorezca el desarrollo de distintas formas de proximidad y procesos de aprendizaje colectivo en colaboración para tener acceso al conocimiento generado en la región. El tema, con muchas aristas aún por analizar, brinda procesos analíticos que facilitan comprender la dinámica propia de estas regiones.

Cabe mencionar que en este estudio se utilizó el concepto de ambiente innovador en países emergentes dadas las características socioeconómicas de México, lo que hace más adecuado emplear esta categoría debido a la presencia de "vacíos estructurales" (Pérez, 2014) en la actividad innovadora que impiden un mayor dinamismo.

De esta manera, al analizar categorías como niveles de proximidad, aprendizaje colectivo y redes, se evidencia el grado en que la región genera, absorbe y explota el conocimiento producido en ella, porque, como se analizó, este es el elemento distintivo que las regiones con ventajas competitivas requieren actualmente.

La dinámica del CIB denota que la instalación de industrias que participan en mercados globales creó un ambiente propicio, sin embargo, aunque se han formado algunas redes para la colaboración academia-industria, aún no se han potenciado en virtud de que cada entidad que participa en el CIB crea incentivos individuales, no regionales, para las empresas y no ha habido estrategias sinérgicas para que esa identidad cultural, que ya existe en la región, se vuelva un factor para la explotación del conocimiento generado.

Los desafíos que el CIB tiene para el futuro están ligados a tres aspectos: el primero, la necesidad de una coordinación de la región para diseñar programas y políticas que atraigan inversiones que favorezcan el desarrollo no solo económico sino también tecnológico para todo el tejido productivo; segundo, la sustentabilidad, dado que algunas ciudades muestran ya un menor crecimiento, por lo que la relocalización hacia áreas periféricas es un elemento que impacta en la atracción de inversiones futuras y tercero, la competencia internacional cada vez más fuerte por aproximar inversiones de sectores de alta tecnología.

\section{Referencias bibliográficas}

ALLEN, J. \& STORPER, M. Regional Development reconsidered. Los Ángeles: Working paper $N^{\circ} 1$, Lewis Center for regional policy Studies University of California, 1990, p. 56.

ALVA, C.; BIZBERG, I. y RIVIERE, H. Las regiones ante la globalización. México: Ed. CEMCA-OSTROM-El Colegio de México, 1988. 
AMBOS, T. \& AMBOS, B. The impact of distance on knowledge transfer effectiveness in multinational corporations. Journal of International Management, 2009, Vol. 15, p. 1-14.

ASHEIM, B. \& COENEN, L. Knowledge bases and regional innovation systems: Comparing Nordic clusters. Research Policy, 2005, Vol. 34, N 8, p. 1173-1190.

BLANCO, M.; PARRA, A. y RUIZ, E. Breve Historia de Guanajuato. México: Fondo de Cultura Económica-Colegio de México, 2000.

BOTTAZZI, G.; DOSI, G. \& FAGIOLO, G. On sectoral specificities in the geography of corporate location. In: BRESCHI, S. \& MALERBA, F. (editors). Clusters, Networks and Innovation. Oxford: Oxford University Press, 2005, p. 54-79.

CABRERO, E. Acción pública y desarrollo local. México: Fondo de Cultura Económica, 2005.

CAMACHO, F. La ciudad de Aguascalientes en la red global: retos para el siglo XXI. En: ARCE, C.; CABRERO, E. y ZICCARDI, A. (coordinadores). Ciudades del Siglo XXI: ¿Competitividad o cooperación? México: CIDE-Miguel Ángel Porrúa, 2005, p. 555-608.

CAMAGNI, R. Introduction: from the local 'mileu' to innovation through cooperation networks. In: CAMAGNI, R. (editor). Innovation networks: spatial perspectives, London/ New York: Belhaven Press, 1991, p. 1-9.

CASALET, M. y GONZÁLEZ, L. El entorno institucional y la formalización de las redes en el sector electrónico de Chihuahua. En: VILLAVICENCIO, D. (coordinador). La emergencia de dinámicas institucionales de apoyo a la industria maquiladora de México. México: Cámara de Diputados-UAM-Miguel Ángel Porrúa, 2006, p. 49-87.

CASAS, R. y LUNA, M. Espacios emergentes de conocimiento en las regiones: hacia una taxonomía. En: CASAS, R. (coordinador). La formación de redes de conocimiento. Una perspectiva Regional desde México. Barcelona: IIS UNAM-Anthropos Editorial, 2001, p. 35-78.
COMISIÓN ECONÓMICA PARA AMÉRICA LATINA Y EL CARIBE (CEPAL). Descentralización y desarrollo económico local: una visión general del caso de México. Santiago de Chile: CEPAL, 2000.

CHÁVEZ, A.M. y GUADARRAMA, J. La región Central de México en transición: tendencias económicas y migratorios a finales del milenio. En: Procesos metropolitanos y grandes ciudades. México: Instituto de Geografía, UNAM, 2003.

CONACYT. Informe General de la Ciencia y Tecnología en México. México: Conacyt, 2012.

CREVOISIER, O. \& MAILLAT, D. Mileu, industrial organization and territorial production system: towards a new theory of spatial development. In: CAMAGNI, R. Innovation networks: spatial perspectives. London/New York: Belhaven Press, 1991, p. 13-34.

DAVID, P. \& FORAY, D. An introduction to the economy of the knowledge society. Department of Economic Discussion Paper Series, 2001, Vol. 25, № 84.

DIMAGGIO, P. \& POWELL, W. The iron cage revisited: institutional isomorphism and collective rationality in organizational fields. American Sociological Review, 1983, Vol. 48, p. 147-160.

FERNÁNDEZ, V.; VIGIL, J. y SEVAL, M. Explorando la región. Territorios, escalas y racionalidades. Revista de Geografía Norte Grande, 2012, N 51, p. 21-41.

FLORIDA, R. The learning region. In: ACS, Z. (editor). Regional innovation, knowledge and global change. London: Pinter, 2000, p. 231-239.

FORO CONSULTIVO CIENTÍFICO Y TECNOLÓGICO. Estadísticas de los sistemas estatales de innovación. México: Vol. 1 y 2, 2012.

GARCÍA, M. Breve Historia de Querétaro. México: Fondo de Cultura Económica-Colegio de México, 1999. 
GARCÍA, F. La planeación del desarrollo regional en México (1900-2006). Revista Investigaciones geográficas, 2008, № 71, p. 102-121.

GERTLER, M. Tacit knowledge in production systems: how important is geography? In: POLENSKE, K. (editor). The Economic Geography of Innovation. Cambridge: Cambridge University Press, 2007, p. 87-111.

GONZÁLEZ, C. Querétaro: opciones económicas en la región centro. En: ARCE, C.; CABRERO, E. y ZICCARDI, A. (coordinadores). Ciudades del Siglo XXI: ¿Competitividad o cooperación? México: CIDE-Miguel Ángel Porrúa, 2005, p. 521-553.

GONZÁLEZ, S. Guadalajara, ¿una ciudad competitiva? Un análisis del proceso de desarrollo y crecimiento urbano de la Zona Metropolitana de Guadalajara, en el contexto del proceso de globalización económica. Periodo 1990-2000. En: ARCE, C.; CABRERO, E. y ZICCARDI, A. (coordinadores). Ciudades del Siglo XXI: ¿Competitividad o cooperación? México: CIDE-Miguel Ángel Porrúa, 2005, p. 227-269.

INSTITUTO NACIONAL DE ESTADÍSTICA Y GEOGRAFÍA (INEGI). Anuario estadístico y geográfico por entidad federativa 2013. México: INEGI, 2013. Disponible en Internet: http://www.inegi.org.mx/prod_serv/contenidos/espanol/bvinegi/productos/integracion/ pais/aepef/2013/aegpef_2013.pdf

KOMNINOS, N. Intelligent Cities: Innovation, Knowledge Systems and Digital Spaces. London: Spon Press, 2002.

KOSCHATZKY, K. Fundamentos de la economía de redes. Especial enfoque a la innovación. Economía Industrial, 2002, №346, p. 15-26.

KRUGMAN, P. Geografía y Comercio. Barcelona: Antoni Bosh Editor, 1992.

MAILLAT, D. Technology, a key factor for regional development. Ginebra: Saint-Saphorin. 1982.

MARTíNEZ, A.; GARCÍA, A. y MURGUÍA, J. Trayectoria tecnológica, algunos aspectos sociales y culturales: el caso de General Mo- tors Complejo Silao. Memorias del $4^{\circ}$ Congreso Internacional de Sistemas de Innovación y Competitividad, 2009.

MÉNDEZ, R. Geografía Económica: La lógica espacial del capitalismo global. Barcelona: Editorial Ariel, 1997.

MÉNDEZ, R. Crecimiento y crisis en la Región metropolitana de Madrid: significado y contradicciones de la economía del conocimiento. Revista de Geografía Norte Grande, 2012, Vol. 51, p. 43-65.

MORENO, A.; RÍOS, D.; ALVA, B. y MEDINA, R. El sistema de innovación regional de San Luis Potosí: avances y retos. Memorias del 3er Congreso Internacional de Sistemas de Innovación y Competitividad, 2008.

MURÍA, J. Breve historia de Jalisco. México: Fondo de Cultura Económica-El Colegio de México, 1994, p. 177-188.

NARVÁEZ, A. Los comités de planeación del desarrollo estatales y municipales. S/f. Dispnible en Internet: http://www.juridicas. unam.mx/publica/librev/rev/gac/cont/16/pr/ pr38.pdf

ORGANIZACIÓN PARA LA COOPERACIÓN Y EL DESARROLLO ECONÓMICOS (OCDE). Estudios de la OCDE de Innovación Regional: 15 estados mexicanos. París: OCDE. 2009.

OCHOA, A. y SÁNCHEZ, G. Breve historia de Michoacán. México: Fondo de Cultura Económica-El Colegio de México, 2003, p. 235-252.

O'RIAIN, S. Globalization and regional development. In: PIKE, A.; RODRIGUEZ-POSE, A. \& TOMANEY, J. Handbook of local and regional development. New York: Routledge, 2011, p. 15-27.

PALACIOS, J. El concepto de región: la dimensión espacial de los procesos sociales. Revista Interamericana de Planificación, 1993, Vol. 17, No 66, p. 56-68.

PASTOR, M. y MEDINA, R. Sistema de innovación en San Luis Potosí: innovación en la industria metalmecánica. Memorias del 
$4^{\circ}$ Congreso Internacional de Sistemas de Innovación y Competitividad. Guanajuato, México, 2008.

PÉREZ, P. El papel de las Organizaciones Intermedias en la Actividad Innovadora: el caso de México. México: Instituto Politécnico Nacional, 2014.

ROJAS, B.; GÓMEZ SERRANO, J.; REYES RODRÍGUEZ, A.; CAMACHO, S. y REYES SAHAGÚN, C. Breve historia de Aguascalientes. México: Fondo de Cultura Económica-El Colegio de México, 1994.

SAINZ, C. Jalisco en movimiento: las regiones y ciudades como base de su desarrollo. En: PASCUAL, J. y FERNÁNDEZ, A. La gobernanza democrática: un nuevo enfoque para los grandes retos urbanos y regionales. Sevilla: Junta de Andalucía.

SAXENIAN, A. Brain circulation and regional innovation: the Silicon Valley-HsinchuShangahi triangle. In: POLENSKE, K. (editor). The Economic Geography of Innovation. Cambridge: Cambridge University Press, 2007.
SECRETARÍA DE ECONOMÍA. Indicadores de Inversión Extranjera Directa. 2014. Dispponible en Internet: http://www.economia.gob

STOPER, M. The limits to the Globalization. Economic Geography, 1992, Vol. 68, p. 60-93.

TÉLLEZ, C. Modernas localizaciones industriales y urbanización difusa. México: El Colegio de Michoacán, 2009.

UNGER, K.; IBARRA, J. y GARDUÑO, R. Especializaciones reveladas y ventajas competitivas en El Bajío Mexicano. México: Documentos de trabajo $N^{\circ}$ 550, CIDE, 2013.

VÁZQUEZ, A. Endogenous Development: Networking, innovation, institutions and cities. London: Routledge, 2002. 
